Conclusions: Predicting the progression of $\mathrm{MCl}$ to $A D$ or DLB based on neuropsychological profiles is challenging and useful for early therapeutic interventions. More studies are needed, since there are some conflicting findings and, at present, the combination of clinical symptoms with neurocognitive assessment and neuroimaging is the ideal method for the prediction of $\mathrm{MCl}$ progression to various types of dementia.

\title{
502 - Diagnosis of dementia and post-diagnostic support - voice of people with dementia living in Poland
}

Authors:

Maćkowiak, M., Ciułkowicz, M, Duda-Sikuła, M., Szcześniak, D., Rymaszewska, J. on behalf of the COGNISANCE Team

Background:

The existing guidelines for the process of dementia diagnosis and treatment does not necessarily translate into optimal care and support for people with dementia. Such situation can have significant consequences. Misunderstanding of the new life situation as well as the negative perception of the diagnostic process itself are intensified by the limited options of post-diagnostic support. Especially in Poland, where the national dementia strategy has still not been implemented, experiences of people undergoing diagnosis and seeking support should be heard. By giving voice to people with dementia we can value their personal experiences, which due to the existing stigma, may often be overlooked and neglected.

\section{Objective:}

The aim of the study is to examine factors (conditions, barriers, facilitators) to receiving a dementia diagnosis and post-diagnostic support from the perspective of people with dementia living in Poland. The research was conducted as part of the JPND project COGNISANCE.

\section{Methods:}

Ten people with dementia diagnosed within last 24 months were interviewed with the use of selected projective techniques (word associations and third person expressive technique). An inductive qualitative content analysis approach has been implemented to analyse the obtained data.

\section{Results:}

Analysis revealed diverse categories encompassing broad spectrum of conditions, barriers and facilitators to receive a diagnosis and post-diagnostic support. People with dementia were prone to feeling positive emotions despite negative initial reactions during the diagnosis. Positive adaptation to the dementia were highly dependent on the empathetic attitude of the doctor and supportive family. Main needs were related to maintaining the independence, staying active, healthy and being socially connected. Interpersonal factors ensured sense of security and stability crucial for the well-being.

\section{Conclusions:}

Presented results provide important insight into the current experiences of people with dementia and represent guidelines for care providers and policy makers in introducing the optimal solutions in dementia support. 